\title{
Principally unimodular skew-symmetric matrices
}

\author{
André Bouchet ${ }^{1}$, W. H. Cunningham ${ }^{2}$ and J. F. Geelen ${ }^{2}$ \\ 1. Département de Mathématiques et Informatique \\ Université du Maine \\ 72017 Le Mans Cedex, France. \\ 2. Department of Combinatorics and Optimization \\ University of Waterloo \\ Waterloo, Ontario, Canada N2L 3G1
}

May 25, 1996

\begin{abstract}
A square matrix is principally unimodular if every principal submatrix has determinant 0 or \pm 1 . Let $A$ be a symmetric $(0,1)$-matrix, with a zero diagonal. A $P U$-orientation of $A$ is a skew-symmetric signing of $A$ that is $\mathrm{PU}$. If $A^{\prime}$ is a PUorientation of $A$, then, by a certain decomposition of $A$, we can construct every PU-orientation of $A$ from $A^{\prime}$. This construction is based on the fact that the PUorientations of indecomposable matrices are unique up to negation and multiplication of certain rows and corresponding columns by -1 . This generalizes the well-known result of Camion, that if a $(0,1)$-matrix can be signed to be totally unimodular then the signing is unique up to multiplying certain rows and columns by -1 . Camion's result is an easy but crucial step in proving Tutte's famous excluded minor characterization of totally unimodular matrices.
\end{abstract}

\section{Introduction}

A square matrix $A$ is called principally unimodular $(P U)$ if every nonsingular principal submatrix is unimodular (that is, has determinant \pm 1 ). Let $A$ be a symmetric $(0,1)$ matrix, with a zero diagonal, a skew-symmetric signing of $A$ is called an orientation of $A$. We are concerned with the orientations of $A$ that are PU; such orientations are called $P U$-orientations, and were initially introduced in relation to circle graphs $[3,6]$.

Let $A$ be a symmetric $(0,1)$-matrix whose rows and columns are indexed by the set $V$, and let $A^{\prime}$ be a PU-orientation of $A$. We can construct other PU-orientations of $A$ from $A^{\prime}$, for instance, $-A^{\prime}$ is $\mathrm{PU}$, we call this construction negation. Also, for $X \subseteq V$, the matrix $\left.\begin{array}{c|c}A^{\prime}[X] & -A^{\prime}[X, V \backslash X] \\ \hline-A^{\prime}[V \backslash X, X] & A^{\prime}[V \backslash X]\end{array}\right)$ is $\mathrm{PU}$ (where $A[X, Y]$ denotes the submatrix of $A$ indexed by 
the rows $X$ and columns $Y$, and $A[X]$ denotes the principal submatrix $A[X, X])$; this operation is called cut-switching. Collectively, we refer to negation and cut-switching as switching.

It is not, in general, the case that every two PU-orientations of $A$ are equivalent under switching; for instance, we show that the matrix $J_{n}-I_{n}$ has $(n-1) ! / 2$ distinct PUorientations, where $J_{n}$ denotes the $n \times n$ all ones matrix, and $I_{n}$ denotes the $n \times n$ identity. Let $X, Y$ be a partition of $V$ with $|X|,|Y| \geq 2$, we call $(X, Y)$ a split of $A$ if the rank of $A[X, Y]$ is at most 1 , a matrix without a split is called prime. Our main result is:

Theorem 1.1 Let $A$ be a symmetric $(0,1)$-matrix with a zero diagonal. If $A$ is prime then every two $P U$-orientations of $A$ are equivalent under switching.

As a corollary of Theorem 1.1 we derive a formula for the number of PU-orientations of $A$ distinct up to switching, assuming that $A$ has a PU-orientation; this formula is based on a decomposition of $A$ using certain splits.

Theorem 1.1 is a generalization of a theorem about totally unimodular matrices: a matrix $B$ is totally unimodular if and only if the matrix $\left(\begin{array}{c|c}0 & B \\ \hline-B^{T} & 0\end{array}\right)$ is PU.

Theorem 1.2 (Camion [8]) If $A$ is a matrix which can be signed to be totally unimodular then such a signing is unique up to multiplication of certain rows and columns by -1 .

Theorem 1.2 is easy to prove, though it is an important step in proofs of Tutte's famous excluded minor characterization of totally unimodular matrices $[20,21,14]$.

Our proof of Theorem 1.1 gives rise to a polynomial-time algorithm for the following problem: Given a symmetric $(0,1)$-matrix $A$ with a zero diagonal that admits a $P U$ orientation, find a PU-orientation of $A$. Such an algorithm implies that the following problems are algorithmically equivalent, in the sense that $\left(Q_{1}\right)$ is polynomial-time solvable

if and only if $\left(Q_{2}\right)$ is polynomial-time solvable. (This equivalence is used in algorithms that recognize total unimodularity.)

$\left(Q_{1}\right)$ Given a symmetric $(0,1)$-matrix $A$ with a zero diagonal, does $A$ admit a PU-orientation?

$\left(Q_{2}\right)$ Given a skew-symmetric matrix $A$, is $A$ PU?

\section{Delta-matroids}

While delta-matroids do not play a role in the proof of Theorem 1.1, the theorem is naturally described in this setting, so we begin by introducing delta-matroids.

Let $A$ be a square matrix with entries defined over a field $F$, and whose rows and columns are both indexed by $V$. Define $\mathcal{F}_{A}=\{S \subseteq V: A[S]$ is nonsingular $\}$; by convention we assume $\emptyset \in \mathcal{F}_{A}$. If $A$ is either symmetric or skew-symmetric then $\mathcal{F}_{A}$ satisfies the Symmetric Exchange Axiom [4]:

(SEA) For $X, Y \in \mathcal{F}$ and $x \in X \Delta Y$ there exists $y \in X \Delta Y$ such that $X \Delta\{x, y\} \in \mathcal{F}$, 
where $X \Delta Y=(X \backslash Y) \cup(Y \backslash X)$. If $\mathcal{F}$ is a nonempty collection of subsets of $V$ and $\mathcal{F}$ satisfies the (SEA) then $M=(V, \mathcal{F})$ is a delta-matroid (see [1]); delta-matroids arising from symmetric and skew-symmetric matrices are called representable (see [4]). A delta-matroid that can be represented by a skew-symmetric PU-matrix is called regular.

If $A$ is a skew-symmetric matrix, then all sets in $\mathcal{F}_{A}$ have even cardinality. A deltamatroid $(V, \mathcal{F})$ is called even if $\left|F_{1} \Delta F_{2}\right|$ is even for all $F_{1}, F_{2} \in \mathcal{F}$.

Let $M=(V, \mathcal{B})$ be a matroid representable over a field $F$. Here $\mathcal{B}$ is the set of bases of $M$. For a basis $B$ of $M$ define a matrix $A$, whose columns are indexed by the set $V \backslash B$, such that $[I \mid A]$ is a linear representation of $M$ over $F$. If $[I \mid A]$ is a representation of $M$

over the reals and $A$ is totally unimodular then $M$ is regular. Define $A^{\prime}=\left(\begin{array}{c|c}0 & A \\ \hline \pm A^{T} & 0\end{array}\right)$. It can be easily verified that $A^{\prime}$ is $\mathrm{PU}$ if and only if $A$ is totally unimodular, and $\mathcal{F}_{A^{\prime}}=\mathcal{B} \Delta B$, where $\mathcal{B} \Delta B=\left\{B^{\prime} \Delta B: B^{\prime} \in \mathcal{B}\right\}$. It is also easy to show that, for a collection of subsets $\mathcal{F}$ of $V$ and a set $S \subseteq V, \mathcal{F}$ satisfies (SEA) if and only if $\mathcal{F} \Delta S$ satisfies (SEA) (see [7]); this operation is called twisting. Two delta-matroids equivalent under twisting are considered to be equivalent, so representability and regularity in delta-matroids naturally generalize their counterparts in matroids.

As is the case with matroids, regularity seems fundamental in the study of representability.

Theorem 1.3 (Geelen [12]) For an even delta-matroid $M$, the following are equivalent:

(i) $M$ is regular,

(ii) $M$ is representable over every field,

(iii) $M$ is representable over both $G F(2)$ and $G F(3)$.

Our proof of Theorem 1.1 can be generalized to prove the following: Given a 3-connected delta-matroid $M$, any two skew-symmetric $G F(3)$-representations of $M$ are switchingequivalent. For the definition of "3-connectivity", we refer the reader to Bouchet [5]. The requirements for unique $G F(3)$-representability in even delta-matroids are remarkably similar to the requirements for unique $G F(4)$-representability in matroids, see Kahn [17]. In this paper we introduce a tool, called a blocking sequence, for studying splits and prime graphs. Blocking sequences have recently been seen to apply to matroid connectivity, and play a vital role in proving the excluded minor characterization of $G F(4)$-representable matroids of Geelen, Gerards and Kapoor [13].

Tutte's famous characterization of regular matroids (see [20, 21]) has been generalized to delta-matroids arising from symmetric matrices [11]. The present work is motivated by the study of delta-matroids represented by skew-symmetric matrices, and the fact that proofs of Tutte's characterization of regular matroids rely heavily on Theorem 1.2. (See, for example, [14]).

\section{Motivations and applications}

The arguments in this paper are mainly graph theoretic, so we begin by restating the problem in terms of graphs. Throughout this paper all graphs will be assumed to be 
simple. The adjacency matrix of an undirected graph $G=(V, E)$ is the $V$ by $V$ symmetric $(0,1)$-matrix that has a 1 in entry $i, j$ if and only if $i j \in E$. The adjacency matrix of a directed graph $\vec{G}=(V, \vec{E})$ is the $V$ by $V$ skew-symmetric $(0, \pm 1)$-matrix that has a 1 in entry $i, j$ if and only if $i j \in \vec{E}$. A digraph $\vec{G}$ is called an orientation (PU-orientation) of a graph $G$ if the adjacency matrix of $\vec{G}$ is an orientation (PU-orientation) of the adjacency matrix of $G$. For an orientation $\vec{G}$ of $G$, we define the operations of negation, cut-switching and switching for $\vec{G}$ as the result of applying the corresponding operations to the adjacency matrix of $\vec{G}$.

Let $G=(V, E)$ be a graph, and let $X, Y$ be disjoint subsets of $V$. We denote by $[X]$ the set of all distinct pairs of vertices in $X$, and we denote by $[X, Y]$ the set of all pairs of vertices containing an element of $X$ and an element of $Y$. For $S \subseteq E$ we denote by $S[X]$ and $S[X, Y]$ the edge sets $S \cap[X]$ and $S \cap[X, Y]$ respectively. The set $E[X, Y]$ is referred to as a cut of $G$. The graph induced by $X$, denoted $G[X]$, is the graph $(X, E[X])$. For a vertex $v \in V$, we denote by $N_{G}(v)$ the neighbour set of $v$. For a graph $G^{\prime}$ we denote by $V_{G^{\prime}}$ and $E_{G^{\prime}}$ its vertex-set and edge-set.

A split of $G$ is a partition $(X, Y)$ of $V$ such that $|X|,|Y| \geq 2$, and the cut $E[X, Y]$ induces a complete bipartite graph. (Note that not all pairs $x \in X, y \in Y$ need be joined by an edge for $(X, Y)$ to be a split; in fact, if the cut $E[X, Y]$ contains no edges $(X, Y)$ is a split.) A prime graph is a graph without any splits; thus, a graph is prime if and only if its adjacency matrix is prime.

\section{Circle Graphs}
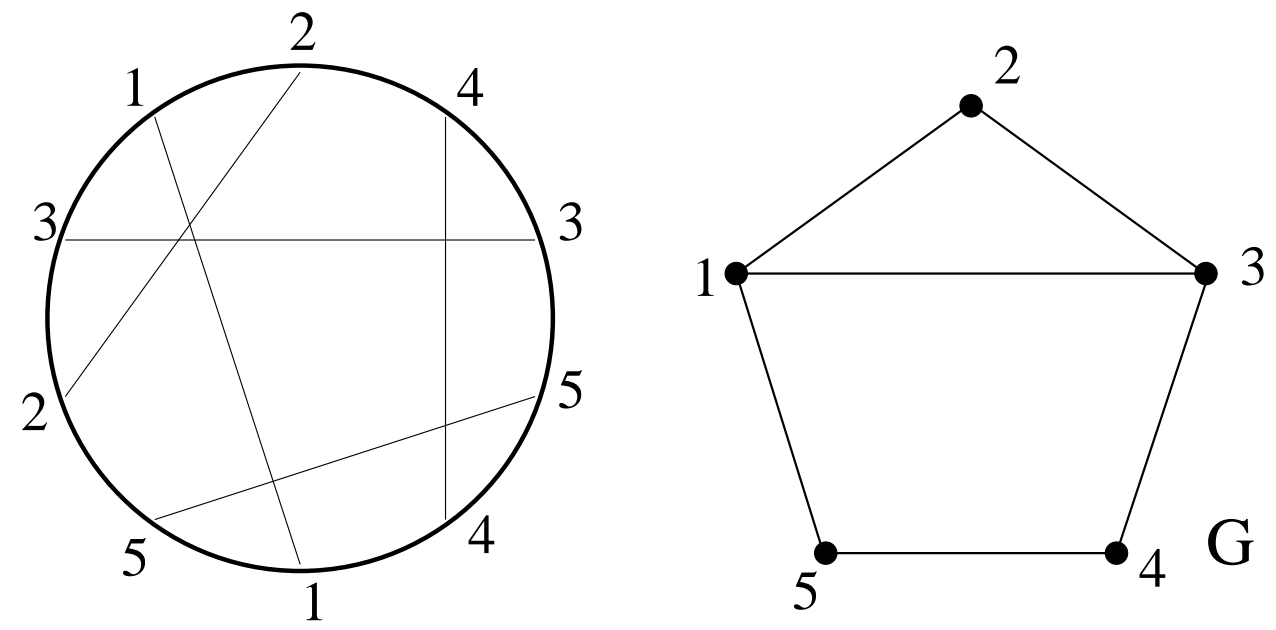

Figure 1: Circle graphs.

In this section we discuss an important class of graphs that admit PU-orientations, namely the circle graphs. A circle graph is the intersection graph of a finite set of chords of a circle. (See Figure 1.) De Fraysseix [10], showed that the bipartite circle graphs are the fundamental graphs of planar graphs. (If $T$ is a spanning tree of a connected graph $G$ then a fundamental graph of $G$ is a bipartite graph with bipartition $E_{T}, E_{G} \backslash E_{T}$ and edges ef where $e \in E_{T}, f \in E_{G} \backslash E_{T}$ and $T+f-e$ is a tree.) It is well known that the fundamental 
matrices (that is, the adjacency matrices of fundamental graphs) of any graph can be signed to be totally unimodular. Hence bipartite circle graphs admit PU-orientations. In fact, this result extends to all circle graphs.
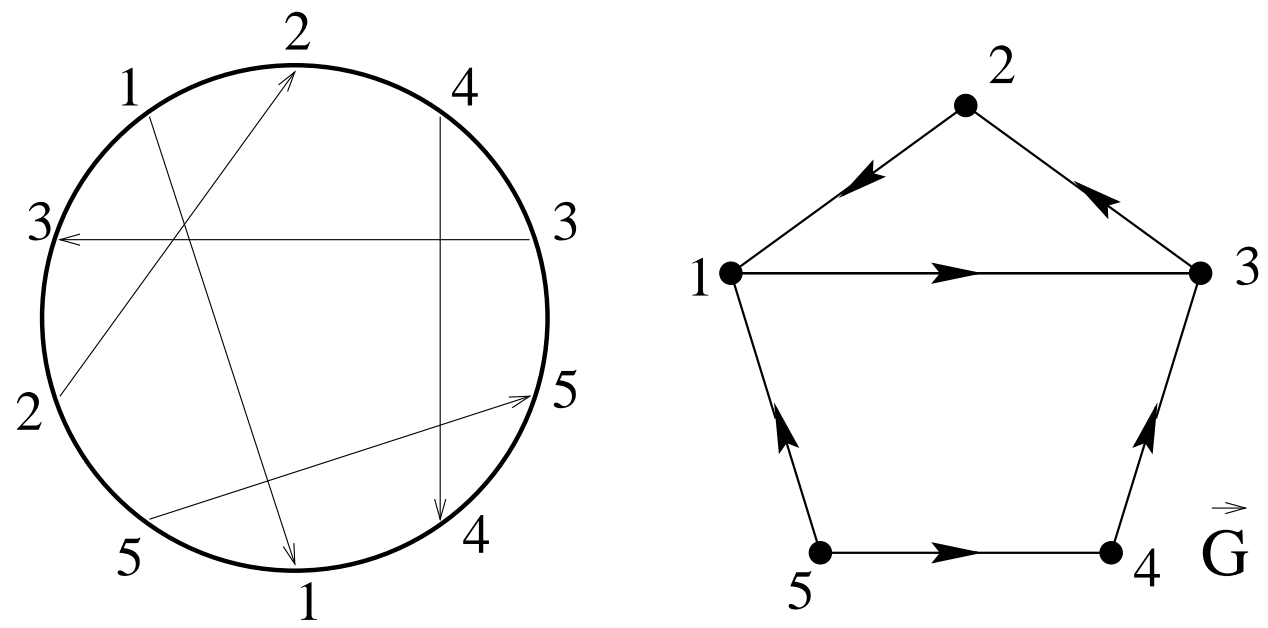

Figure 2: Orienting circle graphs.

Let $G=(V, E)$ be a circle graph represented by a set $V$ of chords of a circle. By possibly perturbing the representation, we may assume that no two chords intersect on the circle. Given an arbitrary orientation of the chords, we define an orientation $\vec{G}$ of $G$. Namely, an edge $u v$ of $G$ is oriented with $v$ as its head if and only if the chord $v$ crosses $u$ from left to right (that is, the tail of $v$ is encountered before the head of $u$ when the circle is traversed in the clockwise direction from the tail of $u$ ). Figure 2 depicts an arbitrary orientation of the representation in Figure 1 and the corresponding orientation of the circle graph. $\vec{G}$ is a PU-orientation of $G$. (see $[3,6]$.)
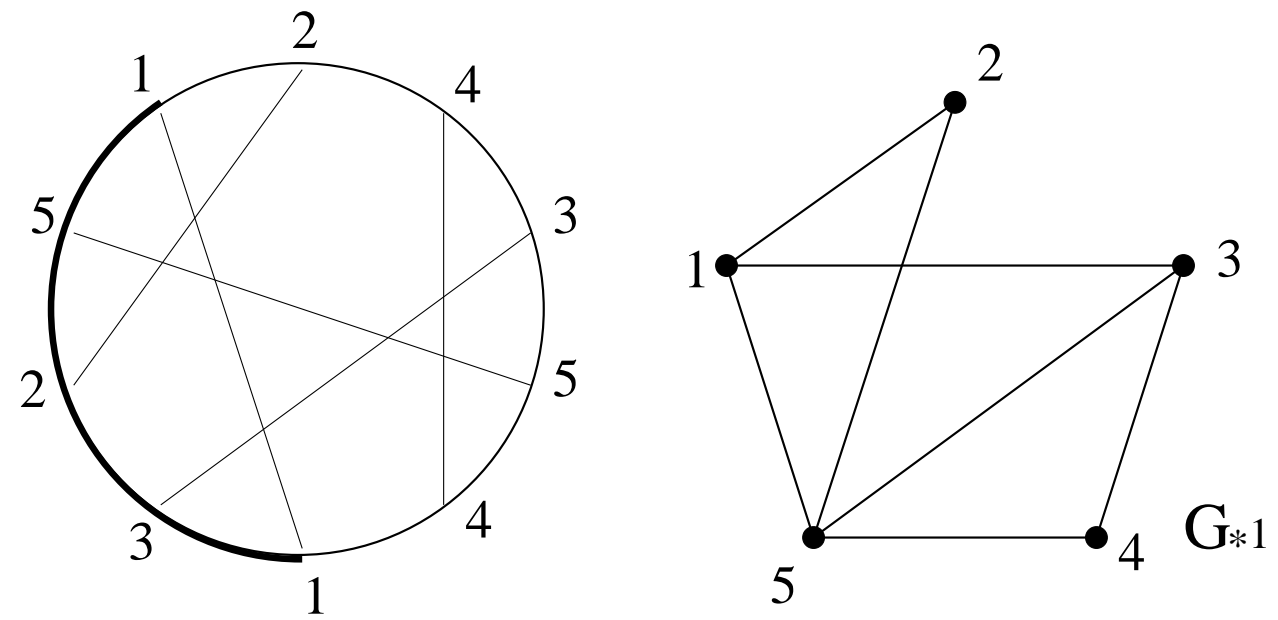

Figure 3: Local complementation.

Given a vertex $v$ of a graph $G$, we define a new graph $G * v$ by complementing the induced graph on the neighbour set of $v$ in $G$; this operation is called local complementation. 
Kotzig [18] noted that $G$ is a circle graph if and only if $G * v$ is a circle graph. Figure 3 demonstrates local complementation on the graph in Figure 1 and the new representation. (In general, if $G$ is a circle graph, then a representation of $G * v$ can be obtained from a representation of $G$ by reversing the order in which chords are encountered while traversing the circle in a clockwise direction from one end of $v$ to the other.) Graphs that admit PU-orientations are not in general preserved under local complementation (in fact, $G$ is a circle graph if and only if every graph equivalent to $G$ under any sequence of local complementations admits a PU-orientation [7]). However, for an edge $u v$ of a graph $G, G$ admits a PU-orientation if and only if $G * u * v * u$ admits a PU-orientation; this operation is called a pivot and will be discussed further in Section 3 .

\section{Decomposition on splits}

Let $G=(V, E)$ be a simple graph, and $(X, Y)$ be a partition of $V$; denote by $G \circ X$ the graph obtained from $G$ by shrinking $X$ to a single vertex, which we label $X$, and removing multiple edges. Similarly if $\vec{G}$ is an orientation of $G$ we define $\vec{G} \circ X$ by shrinking $X$ in $\vec{G}$ and removing multiple arcs. Note that $\vec{G} \circ X$ need not be an orientation of $G \circ X$; however, if the edges in $E[X, Y]$ were all oriented in $\vec{G}$ with their heads in $Y$ then $\vec{G} \circ X$ is an orientation of $G \circ X$. The decomposition $G \circ X, G \circ Y$ of $G$ where $(X, Y)$ is a split was studied in [9]; it has applications in $\Delta$-matroid connectivity [5] and circle graph recognition [2]. Note that $G \circ X$ and $G \circ Y$ are both isomorphic to induced subgraphs of $G$; hence, if $G$ has a PU-orientation then $G \circ X$ and $G \circ Y$ both have PU-orientations. In this section we show that the converse also holds, that is, if $G \circ X$ and $G \circ Y$ both admit PU-orientations then $G$ admits a PU-orientation. Let $\vec{G}_{1}$ and $\vec{G}_{2}$ be PU-orientations of $G \circ X$ and $G \circ Y$ respectively. By cut-switching in $\vec{G}_{1}$, we may assume that no arc in $\vec{G}_{1}$ has $X$ as its head. Similarly, we may assume that no arc in $\vec{G}_{2}$ has $Y$ as its tail. Now construct an orientation $\vec{G}$ of $G$ such that $\vec{G} \circ X=\vec{G}_{1}$ and $\vec{G} \circ Y=\vec{G}_{2} ; \vec{G}$ is called the composition of $\vec{G}_{1}$ and $\vec{G}_{2}$. Before proving that $\vec{G}$ is a PU-orientation, we review some basic results about pfaffians; we use the definition of Stembridge [19].

Let $\vec{G}$ be an orientation of a simple graph $G=(V, E)$, let $A=\left(a_{i j}\right)$ be the adjacency matrix of $\vec{G}$, let $\mathcal{M}_{G}$ denote the set of perfect matchings of $G$, and let $\prec$ be a linear order of $V$. A pair of edges $u_{1} v_{1}, u_{2} v_{2}$ of $G$, where $u_{1} \prec v_{1}$ and $u_{2} \prec v_{2}$, are said to cross if $u_{1} \prec u_{2} \prec v_{1} \prec v_{2}$ or $u_{2} \prec u_{1} \prec v_{2} \prec v_{1}$. (If we place $u_{1}, u_{2}, v_{1}, v_{2}$ on the perimeter of a circle, according to the linear order, then $u_{1} u_{2}$ crosses $v_{1} v_{2}$ if and only if the chords $u_{1} u_{2}$ and $v_{1} v_{2}$ cross.) The sign of a perfect matching $M$ of $G$, denoted $\sigma_{M}$, is $(-1)^{k}$ where $k$ is the number of pairs of crossing edges in $M$. The pfaffian of $A$, denoted $p f(A)$, is defined as follows:

$$
p f(A)=\sum_{M \in \mathcal{M}_{G}} \sigma_{M} \prod_{\substack{u v \in M \\ u \prec v}} a_{u v} .
$$

Surprisingly $p f(A)$ is independent of the order relation; this is reflected by the fundamental identity $\operatorname{det}(A)=p f(A)^{2}$. Like determinants, pfaffians can be calculated by "row 
expansion" [15]:

$$
p f(A)=\sum_{k=2}^{n}(-1)^{k+1} a_{v_{1} v_{k}} p f\left(A\left[V \backslash\left\{v_{1}, v_{k}\right\}\right]\right) \text {, }
$$

where $V=\left\{v_{1}, v_{2}, \ldots, v_{n}\right\}$ and $v_{i} \prec v_{i+1}$, for $i=1,2, \ldots, n-1$.

Proposition 2.1 Let $G$ be a graph containing a split $(X, Y)$. Then the composition of $P U$-orientations of $G \circ X$ and $G \circ Y$ is a $P U$-orientation of $G$.

Proof Let $\vec{G}_{1}$ and $\vec{G}_{2}$ be PU-orientations of $G \circ X$ and $G \circ Y$ respectively, and let $\vec{G}$ be the composition of $\vec{G}_{1}$ and $\vec{G}_{2}$. Let $A, A_{1}$ and $A_{2}$ be the adjacency matrices of $\vec{G}, \vec{G}_{1}$ and $\vec{G}_{2}$ respectively, and let $S \subseteq V$. We are required to prove that $\operatorname{det}(A[S]) \in\{0,1\}$, or equivalently that $p f(A[S]) \in\{0, \pm 1\}$. If $|S \cap X|<2$ or $|S \cap Y|<2$ then $\vec{G}[S]$ is isomorphic to an induced subgraph of $\vec{G}_{1}$ or $\vec{G}_{2}$; hence $\operatorname{det}(A[S]) \in\{0,1\}$. Now, suppose $|S \cap X| \geq 2$ and $|S \cap Y| \geq 2$; then $(X \cap S, Y \cap S)$ is a split in $G[S]$. We assume, without loss of generality, that $V=S$.

Suppose $X=\left\{x_{1}, x_{2}, \ldots, x_{k}\right\}$ and $Y=\left\{y_{1}, y_{2}, \ldots, y_{l}\right\}$. Define a linear order $\prec$ such that

$$
x_{k} \prec x_{k-1} \prec \ldots \prec x_{1} \prec y_{1} \prec y_{2} \prec \ldots \prec y_{l} .
$$

Recall that, for $S \subseteq E_{G}, S[X, Y]$ denotes $S \cap[X, Y]$. Let $\mathcal{M}_{G}^{(i)}=\left\{M \in \mathcal{M}_{G}:|M[X, Y]|=\right.$ $i\}$; then, by (1),

$$
p f(A)=\sum_{i \geq 0} \sum_{M \in \mathcal{M}_{G}^{(i)}} \sigma_{M} \prod_{\substack{u v \in M \\ u<v}} a_{u v}
$$

Claim 1 For $i \geq 2$,

$$
\sum_{M \in \mathcal{M}_{G}^{(i)}} \sigma_{M} \prod_{\substack{u v \in M \\ u \prec v}} a_{u v}=0
$$

Proof of claim: For each matching $M \in \mathcal{M}_{G}^{(i)}$, we define another matching $M^{\prime}$ as follows: choose edges $x_{i_{1}} y_{j_{1}}$ and $x_{i_{2}} y_{j_{2}}$, where $i_{1}<i_{2}$, such that

$$
M\left[\left\{x_{1}, x_{2}, \ldots, x_{i_{2}}\right\}, Y\right]=\left\{x_{i_{1}} y_{j_{1}}, x_{i_{2}} y_{j_{2}}\right\}
$$

then define

$$
M^{\prime}=M \Delta\left\{x_{i_{1}} y_{j_{1}}, x_{i_{2}} y_{j_{2}}, x_{i_{1}} y_{j_{2}}, x_{i_{2}} y_{j_{1}}\right\}
$$

Note that $M=\left(M^{\prime}\right)^{\prime}$, and

$$
\sigma_{M} \prod_{\substack{u v \in M \\ u \prec v}} a_{u v}=-\sigma_{M^{\prime}} \prod_{\substack{u \in M^{\prime} \\ u \prec v}} a_{u v},
$$

which proves the claim.

For any perfect matching $M$ of $G$ we have $|M[X, Y]| \equiv|X|(\bmod 2)$; this gives rise to two cases. 
Case 1: $\quad|X|$ is even. Thus

$$
\begin{aligned}
& p f(A)=\sum_{M \in \mathcal{M}_{G}^{(0)}} \sigma_{M} \prod_{\substack{u v \in M \\
u<v}} a_{u v} \\
& =\sum_{M_{X} \in \mathcal{M}_{G}[X]} \sum_{M_{Y} \in \mathcal{M}_{G}[Y]} \sigma_{M_{X} \cup M_{Y}} \prod_{\substack{u \in \in M_{X} \cup M_{Y} \\
u \prec v}} a_{u v} \\
& =\left(\sum_{M_{X} \in \mathcal{M}_{G}[X]} \sigma_{M_{X}} \prod_{\substack{u v \in M_{X} \\
u \prec v}} a_{u v}\right)\left(\sum_{M_{X} \in \mathcal{M}_{G}[X]} \sigma_{M_{X}} \prod_{\substack{u v \in M_{X} \\
u \prec v}} a_{u v}\right) \\
& =p f(A[X]) p f(A[Y]) \text {. }
\end{aligned}
$$

However $A[X]=A_{2}[X]$ and $A[Y]=A_{1}[Y]$, and $A_{1}$ and $A_{2}$ are PU, so $p f(A)=0, \pm 1$.

Case 2: $\quad|X|$ is odd. Thus

$$
p f(A)=\sum_{M \in \mathcal{M}_{G}^{(1)}} \sigma_{M} \prod_{\substack{u v \in M \\ u \prec v}} a_{u v} .
$$

Every matching $M \in \mathcal{M}_{G}^{(1)}$ can be expressed as $M_{1} \cup M_{2} \cup\left\{x_{i} y_{j}\right\}$, where $M_{1} \in \mathcal{M}_{G\left[X-x_{i}\right]}$ and $M_{2} \in \mathcal{M}_{G\left[Y-y_{i}\right]}$. The set of edges of $M$ that cross $x_{i} y_{j}$ is

$$
M_{1}\left[\left\{x_{1}, \ldots, x_{i-1}\right\},\left\{x_{i+1}, \ldots, x_{k}\right\}\right] \cup M_{2}\left[\left\{y_{1}, \ldots, y_{j-1}\right\},\left\{y_{j+1}, \ldots, y_{l}\right\}\right] ;
$$

furthermore

$$
\begin{aligned}
\left|M_{1}\left[\left\{x_{1}, \ldots, x_{i-1}\right\},\left\{x_{i+1}, \ldots, x_{k}\right\}\right]\right| & \equiv i-1 & & (\bmod 2) \text { and } \\
\left|M_{2}\left[\left\{y_{1}, \ldots, y_{j-1}\right\},\left\{y_{j+1}, \ldots, y_{l}\right\}\right]\right| & \equiv j-1 & & (\bmod 2) .
\end{aligned}
$$

Therefore $\sigma_{M}=\left((-1)^{i-1} \sigma_{M_{1}}\right)\left((-1)^{j-1} \sigma_{M_{2}}\right)$, and

$$
\begin{aligned}
& p f(A)= \sum_{i=1}^{k} \sum_{j=1}^{l} \sum_{M_{1} \in \mathcal{M}_{G\left[X-x_{i}\right]}} \sum_{M_{2} \in \mathcal{M}_{G\left[Y-y_{i}\right]}}\left((-1)^{i-1} \sigma_{M_{1}}\right)\left((-1)^{j-1} \sigma_{M_{2}}\right) \\
& a_{x_{i} y_{j}}\left(\prod_{\substack{u v \in M_{1} \\
u \prec v}} a_{u v}\right)\left(\prod_{\substack{u v \in M_{2} \\
u \prec v}} a_{u v}\right) \\
&=\left(\sum_{i=1}^{k}(-1)^{i+1} \sum_{M_{1} \in \mathcal{M}_{G\left[X-x_{i}\right]}} \sigma_{M_{1}} \prod_{\substack{u v \in M_{1} \\
u \prec v}} a_{u v}\right) \\
&\left(\sum_{j=1}^{l}(-1)^{j+1} \sum_{M_{2} \in \mathcal{M}_{G\left[Y-y_{i}\right]}}^{\sigma_{M_{2}} \prod_{\substack{u v \in M_{2} \\
u \prec v}} a_{u v}}\right) .
\end{aligned}
$$

Now, applying equations (1) and (2),

$$
\begin{aligned}
& p f(A)=\left(\sum_{i=1}^{k}(-1)^{i+1} p f\left(A\left[X-x_{i}\right]\right)\right)\left(\sum_{j=1}^{l}(-1)^{j+1} p f\left(A\left[Y-y_{i}\right]\right)\right) . \\
& p f(A)=-p f\left(A_{1}\right) p f\left(A_{2}\right),
\end{aligned}
$$


and hence, $p f(A) \in\{0, \pm 1\}$, as required.

We remark that there is an alternative proof of Proposition 2.1 that uses pivoting (defined in section 3 ).

\section{Counting PU-orientations}

Let $G=(V, E)$ be a graph with a PU-orientation, and define $\alpha(G)$ to be the number of PU-orientations of $G$ distinct up to cut-switching. Camion's theorem tells us that if $G$ is bipartite then $\alpha(G)=1$; the main result of this paper implies that if $G$ is prime, but not bipartite, then $\alpha(G)=2$. In this section we describe how $\alpha(G)$ can be computed by a canonical decomposition of graphs into graphs that are either prime, bipartite, or complete.

Let $\vec{G}$ be an orientation of $G$, and let $C$ be an even circuit of $G$. We say that $\vec{G}$ is even $(o d d)$ on $C$ if, while traversing $C$ in an arbitrary direction, the number of edges of $C$ that are oriented in the forward direction by $\vec{G}$ is even (odd). Because $C$ has an even number of edges this definition is independent of the direction in which we traverse $C$.

Lemma 2.2 Let $C$ be the circuit $x_{1}, x_{2}, x_{3}, x_{4}, x_{1}$ of a graph $G$, and let $\vec{G}$ be a PUorientation of $G$ that is odd on $C$. Then $G\left[\left\{x_{1}, x_{2}, x_{3}, x_{4}\right\}\right]$ is a complete graph and $\vec{G}$ is even on the circuit $x_{1}, x_{2}, x_{4}, x_{3}, x_{1}$.

Proof This follows by an easy pfaffian calculation, which is left to the reader.

Let $\left(X_{1}, X_{2}\right)$ and $\left(Y_{1}, Y_{2}\right)$ be splits of $G$. We say that $\left(X_{1}, X_{2}\right)$ and $\left(Y_{1}, Y_{2}\right)$ cross if $X_{i} \cap Y_{j} \neq \emptyset$ for each $i, j$; we call the split $\left(X_{1}, X_{2}\right)$ good if there are no splits of $G$ that cross $(X, Y)$. We recursively define a decomposition of a graph $G$ as follows.

- $D=\{H: H$ a connected component of $G\}$ is a decomposition of $G$,

- If $D$ is a decomposition of $G$ and $H \in D$ has a good split $(X, Y)$ then $(D \backslash H) \cup\{H \circ$ $X, H \circ Y\}$ is a decomposition of $G$.

We call the elements of a decomposition $D$ the $D$-components.

Theorem 2.3 If $D$ is a decomposition of $G$ then $\alpha(G)=\prod_{H \in D} \alpha(H)$.

Proof It is clear that $\alpha(G)$ is the product, taken over all connected components $H$ of $G$, of $\alpha(H)$. Thus, it is sufficient to prove that if $(X, Y)$ is a good split of $G$ then $\alpha(G)=\alpha(G \circ X) \alpha(G \circ Y)$. By the composition of PU-orientations of $G \circ X$ and $G \circ Y$, we have that $\alpha(G) \geq \alpha(G \circ X) \alpha(G \circ Y)$. Therefore, it suffices to show that every PUorientation $\vec{G}$ of $G$ is equivalent under cut-switching to a composition of PU-orientations of $G \circ X$ and $G \circ Y$ (that is, $\vec{G}$ can be reoriented by cut-switching so that every edge in $E(X, Y)$ is oriented with its head in $Y)$. Suppose, by way of contradiction, that $\vec{G}$ is a PU-orientation of $G$, and that $\vec{G}$ is not the composition of PU-orientations of $G \circ X$ and $G \circ Y$.

Let $X^{\prime}=N_{G}(Y)$ and $Y^{\prime}=N_{G}(X)$. Choose $x_{1} \in X^{\prime}$ and $y_{1} \in Y^{\prime}$; then, for all $y \in Y^{\prime}$ and $x \in X^{\prime}$, use cut-switching so that the edge $x_{1} y$ is oriented with $x_{1}$ as the tail, and the edge $x y_{1}$ is oriented with $y_{1}$ as the head in $\vec{G}$. Since $\vec{G}$ is not the composition of PUorientations of $G \circ X$ and $G \circ Y$, there exists an edge $x_{2} y_{2}$ of $G$, where $x_{2} \in X^{\prime}$ and $y_{2} \in Y^{\prime}$, 
that is oriented with $x_{2}$ as its head. Partition $X^{\prime}$ into sets $X_{1}, X_{2}$ such that $x \in X_{1}$ if and only if the edge $x y_{2}$ has $y_{2}$ as its head; similarly, partition $Y^{\prime}$ into sets $Y_{1}, Y_{2}$ such that $y \in Y_{1}$ if and only if the edge $x_{2} y$ has $y$ as its head.

For any $y_{i}^{\prime} \in Y_{i}(i=1,2), \vec{G}$ is odd on the circuit $x_{1}, y_{1}^{\prime}, x_{2}, y_{2}^{\prime}, x_{1}$, so, by Lemma 2.2 , $G\left[\left\{x_{1}, x_{2}, y_{1}^{\prime}, y_{2}^{\prime}\right\}\right]$ is a complete graph. Therefore $y_{1}^{\prime} y_{2}^{\prime}$ is an edge of $G$. We similarly prove that $x_{1}^{\prime} x_{2}^{\prime}$ is an edge of $G$ for any $x_{i}^{\prime} \in X_{i}(i=1,2)$. Hence $\left(X_{1} \cup Y_{1}, X_{2} \cup Y_{2}\right)$ is a split of $G\left[X_{1} \cup X_{2} \cup Y_{1} \cup Y_{2}\right]$. However, since $(X, Y)$ is a good split, there cannot exist a split $\left(X^{\prime \prime}, Y^{\prime \prime}\right)$ with $X_{1}, Y_{1} \subseteq X^{\prime \prime}$ and $X_{2}, Y_{2} \subseteq Y^{\prime \prime}$. Therefore, there exists a chordless path $v_{1}, \ldots, v_{p}$ in $V \backslash\left(X^{\prime} \cup Y^{\prime}\right)$ such that $N_{G}\left(v_{i}\right) \cap\left(X_{1} \cup Y_{1}\right) \neq \emptyset$ if and only if $i=1$, and $N_{G}\left(v_{j}\right) \cap\left(X_{2} \cup Y_{2}\right) \neq \emptyset$ if and only if $j=p$. Since $(X, Y)$ is a split in $G,\left\{v_{1}, \ldots, v_{p}\right\}$ is a subset of either $X$ or $Y$; we assume, by possibly exchanging the roles of $X$ and $Y$, that $\left\{v_{1}, \ldots, v_{p}\right\}$ is a subset of $Y$. Choose $y_{1}^{\prime} \in Y_{1}$ adjacent to $v_{1}$, and choose $y_{2}^{\prime} \in Y_{2}$ adjacent to $v_{p}$. Let $H$ be the graph induced by $\left\{x_{1}, x_{2}, y_{1}^{\prime}, y_{2}^{\prime}, v_{1}, \ldots, v_{p}\right\}$; this is depicted by Figure 4 .

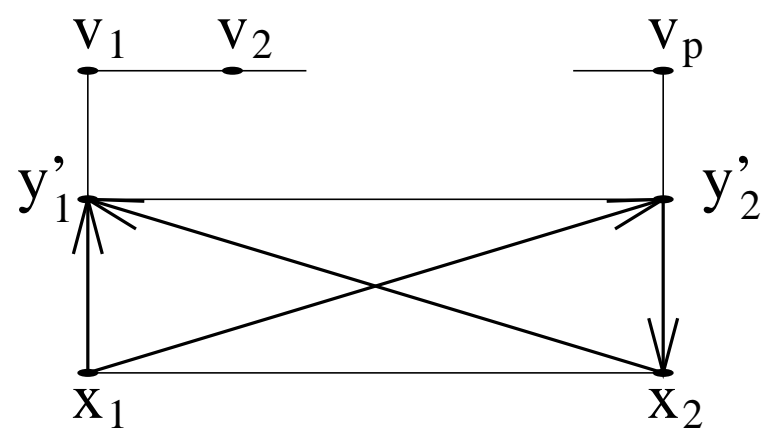

Figure 4: $H$

We assume that $p=1$ or 2 , since otherwise we shorten the path $y_{1}^{\prime}, v_{1}, v_{2}, \ldots, v_{p}, y_{2}^{\prime}$ by pivoting on $v_{1} v_{2}$, and then deleting $v_{1}$ and $v_{2}$ from $G$. If $p=1$ then $\vec{G}$ is odd on exactly one of the circuits $v_{1}, y_{1}^{\prime}, x_{1}, y_{2}^{\prime}, v_{1}$ and $v_{1}, y_{1}^{\prime}, x_{2}, y_{2}^{\prime}, v_{1}$, which, by Lemma 2.2, contradicts that $v_{1}$ is adjacent to neither $x_{1}$ nor $x_{2}$. If $p=2$ then pivoting on $v_{1} v_{2}$ deletes the edge $y_{1}^{\prime} y_{2}^{\prime}$ while leaving $\vec{G}$ odd on the circuit $x_{1}, y_{1}^{\prime}, x_{2}, y_{2}^{\prime}, x_{1}$, contradicting Lemma 2.2 .

Lemma 2.4 For every integer $n, \alpha\left(K_{n}\right)=(n-1)$ !, where $K_{n}$ is the complete graph on $n$ vertices.

Proof Let $\vec{K}_{n}$ be a PU-orientation of $K_{n}$, and let $v$ be any vertex of $K_{n}$. There exists a unique orientation equivalent under cut-switching to $\vec{K}_{n}$ with the property that every edge incident with $v$ has $v$ as its tail; we assume that $\vec{K}_{n}$ has this property.

Suppose that $\vec{K}_{n}$ has a directed circuit, and let $\vec{C}$ be a shortest directed circuit. $\vec{C}$ must have length 3 , since otherwise there exists a chord $e$ of $\vec{C}$ and $\vec{C}+e$ contains a directed circuit shorter than $\vec{C}$. Let $X$ be the vertex set of $\vec{C} . \vec{K}_{n}$ is odd on every circuit of length 4 in $K_{n}[X+v]$, which contradicts Lemma 2.2. Hence $\vec{K}_{n}$ contains no directed circuits. We call such an orientation transitive.

There are $(n-1)$ ! transitive orientations of $K_{n}-v$; thus, $\alpha\left(K_{n}\right) \leq(n-1)$ !, with equality only if every transitive orientation of $K_{n}$ is PU. Every two transitive orientations are isomorphic, so we may assume that $V_{K_{n}}=\{1, \ldots, n\}$, and for $1 \leq i<j \leq n$, the edge $i, j$ is oriented with $j$ as its head in $\vec{K}_{n}$. We have that $\vec{K}_{3}$ is $\mathrm{PU}$; and, for $n>3, K_{n}$ is 
the composition of transitive orientations of two smaller complete graphs. Therefore, by Proposition 2.1 and induction, $\vec{K}_{n}$ is PU.

A decomposition $D$ is called a total decomposition if no $D$-component has a good split. A star graph with $n$ vertices is a graph containing a vertex that is adjacent to $n-1$ vertices of degree 1. Total decompositions were introduced in [9], though our definition of the term decomposition differs slightly from the original definition.

Theorem 2.5 (Cunningham [9]) Let $G$ be a graph. Then

- All total decompositions of $G$ are essentially the same; specifically, if $D_{1}$ and $D_{2}$ are total decompositions of $G$, then there exists a bijection $\pi: D_{1} \rightarrow D_{2}$ such that, for each $D_{1}$-component $H, H$ and $\phi(H)$ are isomorphic.

- If $D$ is the total decomposition of $G$ then every $D$-component is a complete graph, a star graph, or a prime graph.

- The total decomposition can be found in polynomial time.

Let $D$ be the total decomposition of a graph $G$. By Theorem 2.5, every $D$-component $H$ is either complete, prime or bipartite; so, assuming that $G$ has a PU-orientation, we know $\alpha(H)$. Therefore, by Theorem 2.3 , we know $\alpha(G)$ explicitly.

\section{$3 \quad$ Prime graphs}

This section contains the proof of Theorem 1.1. We begin by introducing preliminary results.

\section{Pivoting}

Let $A=\left(a_{i j}\right)$ be a skew-symmetric $(0, \pm 1)$-matrix whose rows and columns are indexed by $V$. Suppose $u, w \in V$ and $a_{u w}=1$. Define $x, y$ so that

$$
A=\left(\begin{array}{c|c|c}
0 & 1 & x^{T} \\
\hline-1 & 0 & y^{T} \\
\hline-x & -y & A[V-u-w]
\end{array}\right)
$$

where the first and second row are indexed by $u$ and $w$ respectively. Then define a matrix $A^{\prime}$ whose rows and columns are also indexed by the set $V$ as follows:

$$
A^{\prime}=\left(\begin{array}{c|c|c}
0 & 1 & y^{T} \\
\hline-1 & 0 & x^{T} \\
\hline-y & -x & A[V-u-w]-y x^{T}+x y^{T}
\end{array}\right) .
$$

The operation that constructs $A^{\prime}$ from $A$ is called a pivot on $u w$ in $A$. If in addition we switch the labels $u$ and $w$, then we call the operation a partial pivot. The following result implies that the family of PU-matrices is closed under pivoting (and hence also under partial pivoting). 
Proposition 3.1 For $S \subseteq V$, $\operatorname{det} A[S]=\operatorname{det} A^{\prime}[S \Delta\{x, y\}]$.

Proof Since pivoting on $u w$ in $A$ has the same effect on principal submatrices of $A[S \cup$ $\{u, w\}]$ as pivoting on $u w$ in $A[S \cup\{u, w\}]$, we may assume that $S \cup\{u, w\}=V$. Furthermore, since pivoting is an involution, we may assume that $u \in S$. Hence it suffices to prove the following two identities:

$$
\begin{aligned}
\operatorname{det} A[V-w] & =\operatorname{det} A^{\prime}[V-u], \text { and } \\
\operatorname{det} A & =\operatorname{det} A^{\prime}[V-u-w] .
\end{aligned}
$$

Note that $A[V-w]$ and $A^{\prime}[V-u]$ are equivalent under row and column operations. Thus, since the determinant is invariant under row and column operations, we have proved (3). Define

$$
B=\left(\begin{array}{c|c|c}
0 & 1 & x^{T} \\
\hline-1 & 0 & y^{T} \\
\hline \underline{0} & \underline{0} & A[V-u-w]-y x^{T}+x y^{T}
\end{array}\right) .
$$

$B$ is obtained from $A$ by row elimination, so $\operatorname{det} A=\operatorname{det} B$; furthermore

$$
\operatorname{det} B=\operatorname{det} B[V-u-w]=\operatorname{det} A^{\prime}[V-u-w] .
$$

Thus we have proved (4).

For a pair $S, S^{\prime}$ of subsets of $V$, if $S$ and $S^{\prime}$ are disjoint, we have defined $\left[S, S^{\prime}\right]=\left\{s s^{\prime}\right.$ : $\left.s \in S, s^{\prime} \in S^{\prime}\right\}$; for intersecting sets $S, S^{\prime}$ we define

$$
\left[S, S^{\prime}\right]=\left[S \backslash S^{\prime}, S^{\prime} \backslash S\right] \cup\left[S \backslash S^{\prime}, S \cap S^{\prime}\right] \cup\left[S^{\prime} \backslash S, S \cap S^{\prime}\right] .
$$

We can interpret partial pivoting over the binary field as a transformation of an undirected graph. Let $G=(V, E)$ be the graph whose adjacency matrix is equivalent to $A$ over $G F(2)$. Define a graph $G^{\prime}=\left(V, E^{\prime}\right)$ where

$$
E^{\prime}=E \Delta\left[N_{G}(u)-w, N_{G}(w)-u\right] .
$$

It is easily verified that the adjacency matrix of $G^{\prime}$ is obtained by performing a partial pivot on $u w$ in $A$ over $G F(2)$.

A consequence of Proposition 3.1 is that pivoting (or partial pivoting) on a PU-matrix yields a $(0, \pm 1)$-matrix. Thus we can think of pivoting and partial pivoting as operations on oriented graphs. Suppose $A$ is $\mathrm{PU}$ and let $\vec{G}=(V, \vec{E})$ be the directed graph having adjacency matrix $A$. Let $\vec{G}^{\prime}=\left(V, \vec{E}^{\prime}\right)$ be the directed graph whose adjacency matrix is obtained by performing a partial pivot on $u w$ (over the reals) in $A$. Then we say that $\vec{G}^{\prime}$ is obtained from $\vec{G}$ by performing a partial pivot on $u w$. Note that the orientation of $u w$ is reversed by the partial pivot. The only other common edges of $G$ and $G^{\prime}$ that may be oriented differently in $\vec{G}$ and $\vec{G}^{\prime}$ are edges whose ends are both common neighbours of $u$ and $w$.

The following result links pivoting and splits; in particular it implies that pivoting preserves prime graphs. It is implied by the fact that local complementation (defined in Section 2) preserves splits (see [2]) and that pivoting on an edge $u w$ of $G$ is equivalent to locally complementing on $u, w, u$ in sequence. 
Proposition 3.2 (Bouchet [5]) Let $(X, Y)$ be a partition of $V$, let $v w \in E$ and let $G^{\prime}=$ $\left(V, E^{\prime}\right)$ be the graph obtained by pivoting on $v w$ in $G$. Then $(X, Y)$ is a split in $G$ if and only if $(X, Y)$ is a split in $G^{\prime}$.

\section{Blocking sequences}

A subsplit of $G$ is a pair $(X, Y)$ of disjoint subsets of $V$ such that $(X, Y)$ is a split in $G[X \cup Y]$ and the cut $E_{G}[X, Y]$ is nonempty. A blocking sequence for the subsplit $(X, Y)$ is a sequence $v_{1}, \ldots, v_{p}$ of vertices in $V \backslash X \backslash Y$ satisfying the following conditions:

1. (a) $\left(X, Y \cup\left\{v_{1}\right\}\right)$ is not a subsplit of $G$,

(b) for all $i<p,\left(X \cup\left\{v_{i}\right\}, Y \cup\left\{v_{i+1}\right\}\right)$ is not a subsplit of $G$, and

(c) $\left(X \cup\left\{v_{p}\right\}, Y\right)$ is not a subsplit of $G$, and

2. no proper subsequence of $v_{1}, \ldots, v_{p}$ satisfies 1 .

We note that the problem of finding a blocking sequence for $(X, Y)$ can be solved by finding a directed path in the digraph $D=D(X, Y)$, with the vertex-set

$$
V(D)=\left\{v_{X}, v_{Y}\right\} \cup(V \backslash X \backslash Y)
$$

and the set of directed edges

$$
\begin{aligned}
E(D)= & \left\{\left(v_{X}, y\right):(X, Y \cup\{y\}) \text { is not a subsplit }\right\} \cup \\
& \{(x, y):(X \cup\{x\}, Y \cup\{y\}) \text { is not a subsplit }\} \cup \\
& \left\{\left(x, v_{Y}\right):(X \cup\{x\}, Y) \text { is not a subsplit }\right\}
\end{aligned}
$$

Then $v_{1}, v_{2}, \cdots, v_{p}$ is a blocking sequence if and only if $v_{X}, v_{1}, v_{2}, \cdots, v_{p}, v_{Y}$ is a directed path with no shortcut in $D$. If no directed path exists in $D$, from $v_{X}$ to $v_{Y}$, then the set

$$
X^{\prime \prime}=\left\{s \in V(D)-v_{X}: \text { a directed path joins } v_{X} \text { to } s\right\}
$$

does not contain $v_{Y}$, and $\left(X^{\prime}, Y^{\prime}\right):=\left(X \cup X^{\prime \prime}, V \backslash X \backslash X^{\prime \prime}\right)$ is a subsplit of $G$.

Proposition 3.3 Let $(X, Y)$ be a subsplit of $G$. There exists a blocking sequence for $(X, Y)$ in $G$ if and only if there exists no split $\left(X^{\prime}, Y^{\prime}\right)$ of $G$ with $X \subseteq X^{\prime}$ and $Y \subseteq Y^{\prime}$.

Proof If there exists a split $\left(X^{\prime}, Y^{\prime}\right)$ of $G$ with $X \subseteq X^{\prime}$ and $Y \subseteq Y^{\prime}$, then $(X \cup\{x\}, Y \cup\{y\})$ is a subsplit for every $x \in X^{\prime} \backslash X$ and $y \in Y^{\prime} \backslash Y$; therefore no blocking sequence exists. Conversely, if no blocking sequence exists, then we can find the required subsplit $\left(X^{\prime}, Y^{\prime}\right)$ by using the digraph $D(X, Y)$.

Following are some results that relate pivoting operations with blocking sequences.

Proposition 3.4 Let $(X, Y)$ be a subsplit of $G$ and let $G^{\prime}$ be a graph obtained by performing a pivot (or partial pivot) on an edge of $G[X]$. A sequence $v_{1}, \ldots, v_{p}$ is a blocking sequence of $(X, Y)$ in $G$ if and only if it is a blocking sequence of $(X, Y)$ in $G^{\prime}$.

Proof Let $X^{\prime}, Y^{\prime}$ be disjoint subsets of $V$ with $X \subseteq X^{\prime}$ and $Y \subseteq Y^{\prime}$. By Proposition 3.2, $\left(X^{\prime}, Y^{\prime}\right)$ is a subsplit of $G^{\prime}$ if and only if it is a subsplit of $G$. The result follows by considering the definition of a blocking sequence. 
Proposition 3.5 Let $v_{1}, \ldots, v_{p}$ be a blocking sequence for a subsplit $(X, Y)$ of $G$, let $x \in$ $X \cap N_{G}\left(v_{1}\right)$ and let $G^{\prime}$ be the graph obtained by performing a partial pivot on the edge $x v_{1}$ in $G$. Suppose that $N_{G}(x) \cap X \neq \emptyset$ and $N_{G}(x) \cap X \neq N_{G}(Y) \cap X$. Then

(i) if $p=1,(X, Y)$ is not a subsplit in $G^{\prime}$, and

(ii) if $p>1, v_{2}, \ldots, v_{p}$ is a blocking sequence for $(X, Y)$ in $G^{\prime}$.

Proof (i) Suppose $p=1$. Let $X^{\prime}=N_{G}(Y) \cap X$ and $Y^{\prime}=N_{G}(X) \cap Y$. Then, since $(X, Y)$ is a subsplit, $E_{G}[X, Y]=\left[X^{\prime}, Y^{\prime}\right]$. Note that

$$
[P, Q] \cap[R, S]=[P \cap R, Q \cap S] \Delta[P \cap R, Q \cap S]
$$

holds for any subsets $P, Q, R$ and $S$ of $V$. Therefore

$$
\begin{aligned}
E_{G^{\prime}}[X, Y] & =\left(E_{G} \Delta\left[N_{G}\left(v_{1}\right)-x, N_{G}(x)-v_{1}\right]\right) \cap[X, Y] \\
& =\left[X^{\prime}, Y^{\prime}\right] \Delta\left[\left(N_{G}\left(v_{1}\right)-x\right) \cap X, N_{G}(x) \cap Y\right] \Delta\left[N_{G}(x) \cap X, N_{G}\left(v_{1}\right) \cap Y\right] .
\end{aligned}
$$

We consider two cases; in each case we use the following fact:

Suppose $E_{G^{\prime}}[X, Y]=\left[X_{1}, Y_{1}\right] \Delta\left[X_{2}, Y_{2}\right]$ where $X_{1}$ and $X_{2}$ are distinct nonempty subsets of $X$, and $Y_{1}$ and $Y_{2}$ are distinct, nonempty subsets of $Y$. Then $(X, Y)$ is not a subsplit in $G^{\prime}$.

Case 1: $\quad x \notin X^{\prime}$. Then $N_{G}(x) \cap Y=\emptyset$, so

$$
E_{G^{\prime}}(X, Y)=\left[X^{\prime}, Y^{\prime}\right] \Delta\left[N_{G}(x) \cap X, N_{G}\left(v_{1}\right) \cap Y\right] .
$$

Furthermore, by the conditions of the proposition, $X^{\prime}, N_{G}(x) \cap X$ are distinct, nonempty subsets of $X$, and, by the definition of a blocking sequence, $Y^{\prime}, N_{G}\left(v_{1}\right) \cap Y$ are distinct, nonempty subsets of $Y$, so $(X, Y)$ is not a subsplit in $G^{\prime}$.

Case 2: $\quad x \in X^{\prime}$. Then $N_{G}(x) \cap Y=Y^{\prime}$. Note that, for any sets $A \subseteq Y, B_{1}, B_{2} \subseteq X$, $\left[A, B_{1}\right] \Delta\left[A, B_{2}\right]=\left[A, B_{1} \Delta B_{2}\right]$, so

$$
E_{G^{\prime}}[X, Y]=\left[X^{\prime} \Delta\left(\left(N_{G}\left(v_{1}\right)-x\right) \cap X\right), Y^{\prime}\right] \Delta\left[N_{G}(x) \cap X, N_{G}\left(v_{1}\right) \cap Y\right] .
$$

Now $x \in X^{\prime} \Delta\left(\left(N_{G}\left(v_{1}\right)-x\right) \cap X\right)$. However $x \notin N_{G}(x) \cap X$, so $X^{\prime} \Delta\left(\left(N_{G}\left(v_{1}\right)-x\right) \cap X\right)$, $N_{G}(x) \cap X$ are distinct, nonempty subsets of $X$. Furthermore, by the definition of a blocking sequence, $Y^{\prime}, N_{G}\left(v_{1}\right) \cap Y$ are distinct nonempty subsets of $Y$; hence $(X, Y)$ is not a subsplit in $G^{\prime}$.

(ii) Suppose $p>1$. By the minimality of a blocking sequence we have that $\left(X, Y \cup\left\{v_{2}\right\}\right)$ is a subsplit in $G$. Note that $v_{1}$ is a blocking sequence for the subsplit $\left(X, Y \cup\left\{v_{2}\right\}\right)$ in $G$. By part (i) of the proposition, $\left(X, Y \cup\left\{v_{2}\right\}\right)$ is not a subsplit in $G^{\prime}$. Also note that $\left(X \cup\left\{v_{1}\right\}, Y\right)$ is a subsplit in $G$ and that $v_{2}, \ldots, v_{p}$ is a blocking sequence for $\left(X \cup\left\{v_{1}\right\}, Y\right)$ in $G$. By Proposition 3.4, $v_{2}, \ldots, v_{p}$ is also a blocking sequence for $\left(X \cup\left\{v_{1}\right\}, Y\right)$ in $G^{\prime}$, and, since $\left(X, Y \cup\left\{v_{2}\right\}\right)$ is not a subsplit in $G^{\prime}, v_{2}, \ldots, v_{p}$ is also a blocking sequence for $(X, Y)$ in $G^{\prime}$. 


\section{Sign-fixed circuits}

Let $C$ be a circuit in a graph $G$. We say that $C$ is sign-fixed with respect to $G$ if any two PU-orientations of $G$ differ on an even number of edges of $C$. For subgraphs $H_{1}, H_{2}$ of $G$, we denote by $H_{1} \Delta H_{2}$ the subgraph of $G$ induced by the edges $E_{H_{1}} \Delta E_{H_{2}}$.

Proposition 3.6 Let $C$ be a circuit of a graph $G$. If there exist sign-fixed circuits $C_{1}, \ldots, C_{k}$ of $G$ such that $C=C_{1} \Delta C_{2} \Delta \ldots \Delta C_{k}$ then $C$ is sign-fixed in $G$.

Proof Let $\vec{G}_{1}, \vec{G}_{2}$ be any pair of PU-orientations of $G$. Let $S$ be the set of edges of $G$ in which the orientations $\vec{G}_{1}$ and $\vec{G}_{2}$ differ. For each sign-fixed circuit $C_{i},\left|C_{i} \cap S\right|$ is even. Now

$$
\begin{aligned}
C \cap S & =\left(C_{1} \Delta \ldots \Delta C_{k}\right) \cap S \\
& =\left(C_{1} \cap S\right) \Delta \ldots \Delta\left(C_{k} \cap S\right) .
\end{aligned}
$$

Since $C \cap S$ can be represented as the symmetric difference of even sets, $C \cap S$ has even cardinality. Hence $C$ is sign-fixed in $G$.

The following proposition is attributed to Bondy in [16]; it can be proved using Menger's theorem.

Proposition 3.7 Let $H$ be an eulerian subgraph of a 2-vertex-connected graph $G$. If $H$ has an even number of edges, then there exist even circuits $C_{1}, \ldots, C_{k}$ of $G$ such that

$$
H=C_{1} \Delta C_{2} \Delta \ldots \Delta C_{k} .
$$

Lemma 3.8 Let $G$ be a graph such that every even circuit is sign-fixed. All PU-orientations of $G$ are switching-equivalent if $G$ is bipartite or 2-connected.

Proof Let $\vec{G}_{1}, \vec{G}_{2}$ be PU-orientations of $G$. If $C^{\prime}$ is an even circuit of $G$, then $\vec{G}_{1}$ and $\vec{G}_{2}$ differ on an even number of edges of $C^{\prime}$, by the premises of the lemma. We claim that the same property may be assumed for every circuit $C^{\prime}$ of $G$. This is obvious if $G$ is bipartite. Otherwise fix an odd circuit $C$. We may assume that the orientations $\vec{G}_{1}$ and $\vec{G}_{2}$ differ on an even number of edges of $C$; otherwise we reverse the orientation $\vec{G}_{2}$. Consider any other odd circuit $C^{\prime}$ of $G$. By Proposition 3.7 there exist even circuits $C_{1}, \ldots, C_{k}$ such that $C^{\prime} \Delta C=C_{1} \Delta \ldots \Delta C_{k}$, therefore $C^{\prime}=C \Delta C_{1} \Delta \ldots \Delta C_{k}$. It follows similarly to the the proof of Proposition 3.6, that the orientations $\vec{G}_{1}$ and $\vec{G}_{2}$ differ on an even number of edges of $C^{\prime}$. Which proves the claim.

Let $S$ be the set of edges upon which the orientations $\vec{G}_{1}$ and $\vec{G}_{2}$ differ. It follows from the claim that if we contract each of the edges in $E_{G} \backslash S$, then we obtain a bipartite graph. Therefore the edges $S$ form a cut in $G$, so $\vec{G}_{1}$ and $\vec{G}_{2}$ are equivalent under cut-switching.

Corollary 3.9 If $G$ is prime and every even circuit of $G$ is sign-fixed, then all $P U$ orientations of $G$ are switching equivalent.

Proof Trivially we may assume $G$ has at least 4 vertices. Then $G$ is 2-connected.

Lemma 3.8 generalizes the ideas used in Seymour's proof of Theorem 1.2. Following is a summary of Seymour's proof. Suppose $C$ is a circuit of a bipartite graph $G$. If $C$ is chordless then it is easy to show that $C$ is sign-fixed. Otherwise, if $C$ has a chord. then $C$ can be expressed as the symmetric difference of two shorter circuits, so inductively we can prove that $C$ is sign-fixed. Then, by Lemma 3.8, all PU-orientations of $G$ are switching-equivalent. 


\section{Decomposition of circuits}

In this section we describe three decompositions of an even circuit $C$ into a symmetric difference of shorter even circuits.

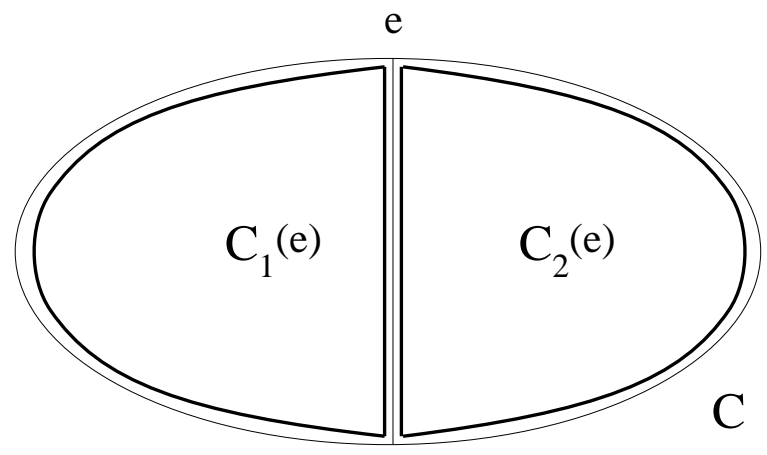

Figure 5: $C+e$

Let $C$ be an even circuit and let $e$ be a chord of $C$. $C$ can be expressed as the symmetric difference of two shorter circuits (see Figure 5) denoted $C_{1}(e), C_{2}(e)$ (in no particular order). Since $C$ is even, $C_{1}(e)$ and $C_{2}(e)$ are either both even or both odd. We say that $e$ is an even (odd) chord of $C$ if $C_{1}(e)$ and $C_{2}(e)$ are both even (odd). The first decomposition of $C$ is $C=C_{1}(e) \Delta C_{2}(e)$, when $e$ is an even chord.

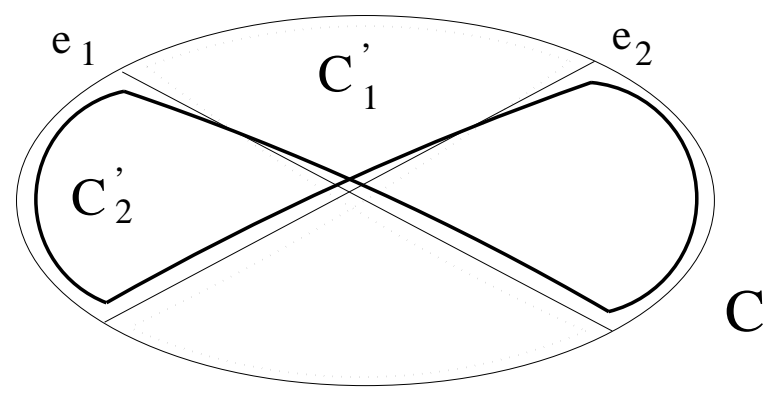

Figure 6: Decomposition of $C+e_{1}+e_{2}$

Let $e_{1}$ and $e_{2}$ be odd chords of an even circuit $C$. We say that $e_{1}$ and $e_{2}$ cross if $e_{1}$ and $e_{2}$ have disjoint ends and $e_{2}$ has exactly one end in $C_{1}\left(e_{1}\right)$. If $e_{1}$ and $e_{2}$ are crossing then define $C_{1}^{\prime}=C_{1}\left(e_{1}\right) \Delta C_{1}\left(e_{2}\right)$ and $C_{2}^{\prime}=C_{1}\left(e_{1}\right) \Delta C_{2}\left(e_{2}\right)$; see Figure $6 . C_{1}^{\prime}$ and $C_{2}^{\prime}$ are both even circuits and

$$
\begin{aligned}
C_{1}^{\prime} \Delta C_{2}^{\prime} & =\left(C_{1}\left(e_{1}\right) \Delta C_{1}\left(e_{1}\right)\right) \Delta\left(C_{1}\left(e_{1}\right) \Delta C_{2}\left(e_{2}\right)\right) \\
& =C_{1}\left(e_{2}\right) \Delta C_{2}\left(e_{2}\right) \\
& =C .
\end{aligned}
$$

If either $C_{1}^{\prime}$ or $C_{2}^{\prime}$ has length 4 then the other has the same length as $C$; otherwise both $C_{1}^{\prime}$ and $C_{2}^{\prime}$ are shorter than $C$. We say that $e_{1}$ and $e_{2}$ are tight crossing chords if either $C_{1}^{\prime}$ or $C_{2}^{\prime}$ has length 4 . The second decomposition of $C$ is $C=C_{1}^{\prime} \Delta C_{2}^{\prime}$, when $e_{1}$ and $e_{2}$ are not tight crossing chords. 
Note that it is not possible to have three odd chords of a circuit such that each pair is a tight crossing pair, so if we have any three mutually crossing odd chords of a circuit $C$, we can apply one of the above decompositions to express $C$ as the symmetric difference of two shorter even circuits.

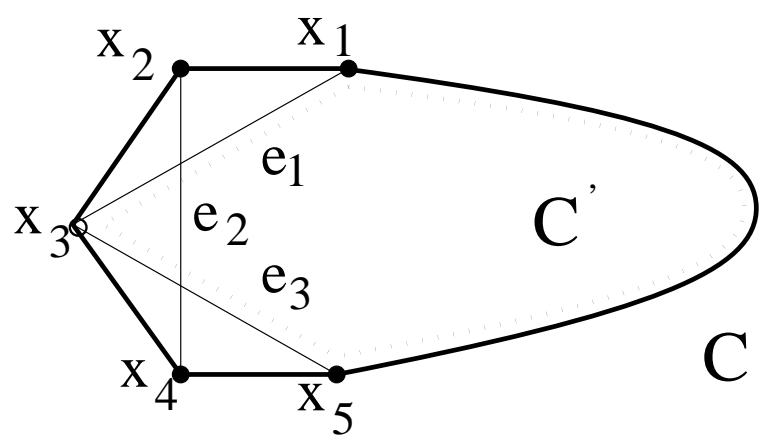

Figure 7: $C+e_{1}+e_{2}+e_{3}$

In the third decomposition we have three odd chords $e_{1}, e_{2}$ and $e_{3}$ of an even circuit $C$ such that $\left\{e_{1}, e_{2}\right\}$ and $\left\{e_{2}, e_{3}\right\}$ are pairs of tight crossing chords and $e_{1}$ and $e_{3}$ do not cross. In this situation there are consecutive vertices $x_{1}, \ldots, x_{5}$ in $C$ such that $e_{1}, e_{2}$ and $e_{3}$ have ends $\left\{x_{1}, x_{3}\right\},\left\{x_{2}, x_{4}\right\}$ and $\left\{x_{3}, x_{5}\right\}$ respectively, as depicted in Figure 7 . Also depicted in Figure 7 is an even circuit $C^{\prime}$; $C$ is the symmetric difference of $C^{\prime}$ and the two circuits $x_{1}, x_{2}, x_{4}, x_{3}, x_{1}$ and $x_{5}, x_{4}, x_{2}, x_{3}, x_{5}$. Furthermore each of these circuits is even and shorter than $C$.

A circuit is said to be decomposable (otherwise indecomposable) if by one of the above decompositions we can express $C$ as the symmetric difference of shorter even circuits. More rigorously, an even circuit $C$ is indecomposable if the chords of $C$ are all odd, each chord crosses at most one other chord and all crossings are tight.

\section{PU-orientations of prime graphs}

We now prove the main result of the paper.

Proof of Theorem 1.1. By Corollary 3.9, it suffices to show that in a prime graph all even circuits are sign-fixed. We prove this by induction on the length of an even circuit. Let $k \geq 4$ be an even integer. We assume that in every prime graph every even circuit of length less than $k$ is sign-fixed.

Let $C^{\prime}$ be a circuit of length $k$ in a prime graph $G^{\prime}$. If $C^{\prime}$ can be expressed as the symmetric difference of sign-fixed circuits in $G^{\prime}$ then, by Proposition 3.6, $C^{\prime}$ is sign-fixed. In particular, if $C^{\prime}$ is decomposable then $C^{\prime}$ is sign-fixed.

Claim 1 Let $C$ be a circuit of length $k$ in a prime graph $G$. If there exists a vertex that has degree 2 in $G\left[V_{C}\right]$ then $C$ is sign-fixed.

Proof of claim In the case that $C$ has length 4, the claim follows from Lemma 2.2. Now suppose that $k>4$ and that $C$ is indecomposable. Let $v$ be a vertex of degree 2 in $G\left[V_{C}\right]$, let $u, w$ be the neighbours of $v$ in $G\left[V_{C}\right]$ and let $G^{\prime}$ be the graph obtained by performing a partial pivot on $v w$ in $G$. 
Let $u^{\prime} u$ and $w w^{\prime}$ be the edges other than $u v$ and $u w$ incident to $u$ and $w$ respectively in $C$. Note that $u^{\prime}$ is not adjacent to $w$ in $G$ since such an edge would be an even chord of $C$, and similarly $u$ is not adjacent to $w^{\prime}$. We have that $N_{G\left[V_{C}\right]}(v)-w=\{u\}$, so

$$
E_{G^{\prime}}\left[V_{C}\right]=E_{G}\left[V_{C}\right] \Delta\left[\{u\}, N_{G\left[V_{C}\right]}(w)-v\right] .
$$

Therefore the partial pivot affects only edges incident with $u$, but the edges $u u^{\prime}$ and $u v$ are unaffected by the partial pivot, so $C$ is a circuit in $G^{\prime}$. Furthermore if the partial pivot were performed on any orientation of $G$, then exactly one edge of $C$, namely $v w$, will be reoriented, so $C$ is sign-fixed in $G$ if and only if $C$ is sign-fixed in $G^{\prime}$. Now $u w^{\prime}$ is an edge of $G^{\prime}$, so $C$ has an even chord in $G^{\prime}$. Hence $C$ is sign-fixed in $G^{\prime}$. This proves Claim 1.

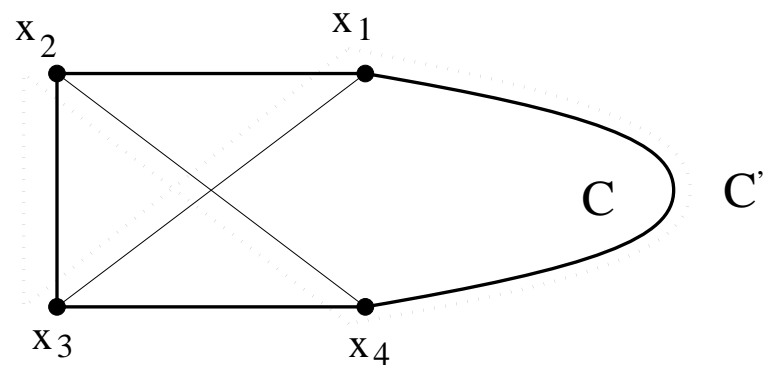

Figure 8: Circuits in Claim 2.

Claim 2 Let $C$ be a circuit of length $k$ in a prime graph $G$, and suppose $x_{1}, \ldots, x_{4}$ are consecutive vertices of $C$ such that $x_{1} x_{3}$ and $x_{2} x_{4}$ are chords of $C$. Finally let $C^{\prime}$ be the symmetric difference of $C$ and the circuit $x_{1}, x_{3}, x_{4}, x_{2}, x_{1}$. (See Figure 8.) Then at least one of $C$ and $C^{\prime}$ is sign-fixed.

Proof of claim The claim is trivially true when $C$ is decomposable, so suppose that $C$ is indecomposable. Let $X=\left\{x_{2}, x_{3}\right\}$ and $Y=V_{C} \backslash X$, and let $e_{1}$ and $e_{2}$ be the edges $x_{1} x_{3}$ and $x_{2} x_{4}$, respectively. Note that $e_{1}$ and $e_{2}$ are crossing chords of $C$, so there are no other chords which cross either $e_{1}$ or $e_{2}$. Hence $(X, Y)$ is a subsplit of $G$; let $v_{1}, \ldots, v_{p}$ be a blocking sequence for this subsplit. We prove the claim by induction on the length of the blocking sequence.

Case 1: $\quad p=1 . v_{1}$ is a blocking sequence for the subsplit $(X, Y)$ in $G$. Then $v_{1}$ is adjacent to exactly one of $x_{2}$ and $x_{3}$. Assume with no loss of generality that $v_{1}$ is adjacent to $x_{2} . v_{1}$ must also be adjacent to some vertex in $Y$. This gives rise to two subcases.

Case 1.1: $\quad v_{1}$ is adjacent to a vertex $y$ in $Y \backslash\left\{x_{1}, x_{4}\right\}$. We assume that $x_{2}$ and $y$ are an even distance apart in $C$. (Otherwise $x_{2}$ and $y$ are an even distance apart in $C^{\prime}$ and we can interchange the roles of $C$ and $C^{\prime}$.) Consider the circuits $C_{1}$ and $C_{2}$ defined by Figure 9 . $C_{1}$ and $C_{2}$ are both even and have length at most $k . x_{3}$ and $x_{2}$ have degree 2 in $G\left[V_{C_{1}}\right]$ and $G\left[V_{C_{2}}\right]$ respectively, so by Claim $1, C_{1}$ and $C_{2}$ are both sign-fixed. Furthermore $C$ is the symmetric difference of $C_{1}$ and $C_{2}$ so $C$ is also sign-fixed. This completes the proof of Claim 2 in Case 1.1.

Case 1.2: $\quad v_{1}$ is not adjacent to any vertices in $Y \backslash\left\{x_{1}, x_{4}\right\}$. In this case $v_{1}$ cannot be adjacent to both $x_{1}$ and $x_{4}$ since otherwise $\left(X \cup\left\{v_{1}\right\}, Y\right)$ would be a subsplit, contradicting the definition of a blocking sequence. So $v_{1}$ is adjacent to exactly one of $x_{1}$ and $x_{4}$. We assume that $v_{1}$ is adjacent to $x_{1}$. (The other case is equivalent under interchanging the 


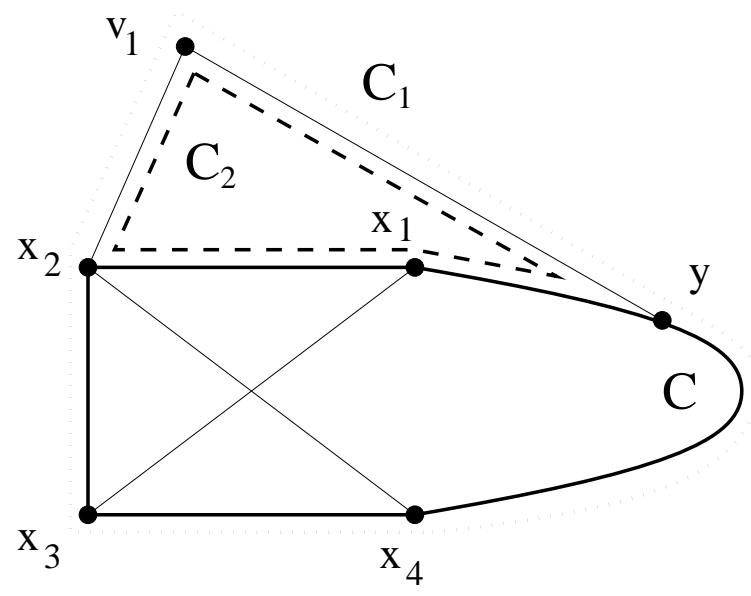

Figure 9: Decomposition in Case 1.1.

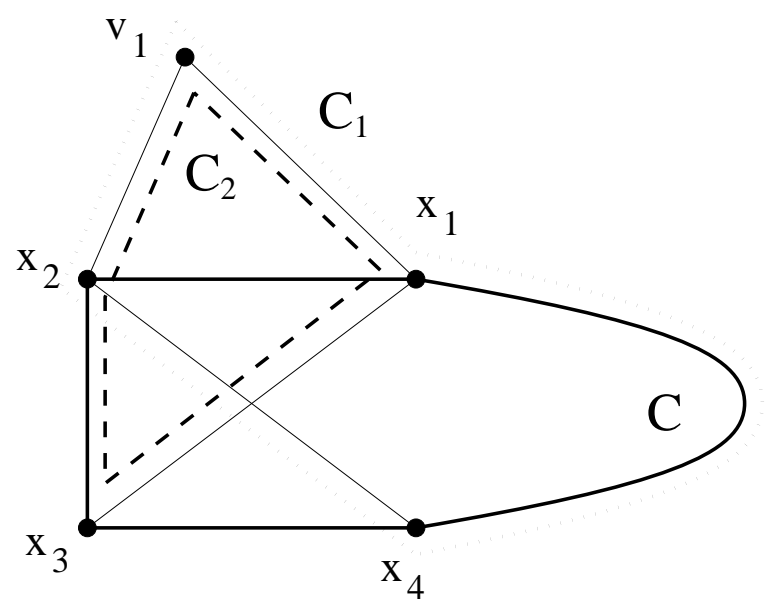

Figure 10: Decomposition in Case 1.2.

roles of $C$ and $C^{\prime}$ and changing labels.) Consider the even circuits $C_{1}$ and $C_{2}$ defined by Figure 10. $v_{1}$ has degree 2 in both $G\left[V_{C_{1}}\right]$ and $G\left[V_{C_{2}}\right]$, so by Claim $1, C_{1}$ and $C_{2}$ are both sign-fixed. $C^{\prime}$ is the symmetric difference of $C_{1}$ and $C_{2}$ so $C^{\prime}$ is also sign-fixed. This completes the proof of Claim 2 in Case 1.

Case 2: $\quad p>1$. As with Case 1, $v_{1}$ is adjacent to exactly one of $x_{2}$ and $x_{3}$, and we assume with no loss of generality that $x_{2}$ and $v_{1}$ are adjacent. $\left(X \cup\left\{v_{1}\right\}, Y\right)$ is a subsplit, so either $N_{G}\left(v_{1}\right) \cap Y=\emptyset$ or $N_{G}\left(v_{1}\right) \cap Y=N_{G}(X) \cap Y=\left\{x_{1}, x_{4}\right\}$. This gives two subcases.

Case 2.1: $\quad N_{G}\left(v_{1}\right) \cap Y=\emptyset$. Let $G^{\prime}$ be the graph defined by performing a partial pivot on the edge $x_{2} v_{1}$. Note that $N_{G}\left(v_{1}\right) \cap V_{C}=\left\{x_{2}\right\}$, so $G\left[V_{C}\right]=G^{\prime}\left[V_{C}\right]$. Then $C$ and $C^{\prime}$ are circuits in $G^{\prime}$ and, by considering the effect of this partial pivot on an orientation of $G, C$ and $C^{\prime}$ are sign-fixed in $G$ if and only if they are sign-fixed in $G^{\prime}$. Now, by Proposition $3.5, v_{2}, \ldots, v_{p}$ is a blocking sequence for the subsplit $(X, Y)$ in $G^{\prime}$, so, by the induction hypothesis of the claim, one of $C$ and $C^{\prime}$ is sign-fixed in $G^{\prime}$.

Case 2.2: $\quad N_{G}\left(v_{1}\right) \cap Y=\left\{x_{1}, x_{4}\right\}$. We have that $v_{2}, \ldots, v_{p}$ is a blocking sequence for the subsplit $\left(X \cup\left\{v_{1}\right\}, Y\right)$. Furthermore, for $i>1,\left(X, Y \cup\left\{v_{i}\right\}\right)$ is a subsplit; it follows 


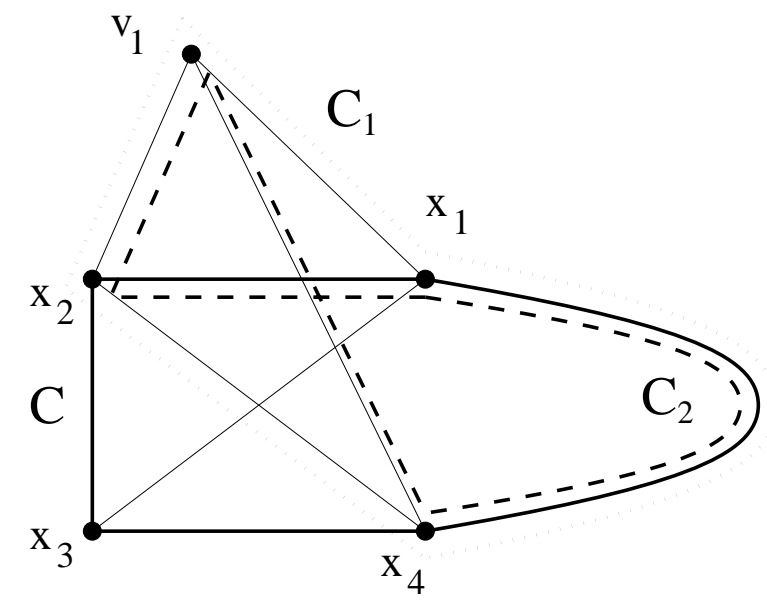

Figure 11: Decomposition in Case 2.2.

that $v_{i}$ is adjacent with $x_{2}$ if and only if $v_{i}$ is adjacent with $x_{3}$. Consequently $v_{2}, \ldots, v_{p}$ is a blocking sequence for the subsplit $\left(\left\{x_{2}, v_{1}\right\}, Y\right)$. Now, by the induction hypothesis of the claim, one of the circuits $C_{1}$ or $C_{2}$, defined in Figure 11, is sign-fixed. Let $C_{1}^{\prime}$ and $C_{2}^{\prime}$ be the circuits $v_{1}, x_{1}, x_{3}, x_{2}, v_{1}$ and $v_{1}, x_{4}, x_{3}, x_{2}, v_{1}$ respectively. $C_{1}^{\prime}$ and $C_{2}^{\prime}$ are both sign-fixed by Claim 1. If $C_{1}$ is sign-fixed then $C^{\prime}$, which is the symmetric difference of $C_{1}$ and $C_{1}^{\prime}$, is sign-fixed. Otherwise $C_{2}$ is sign-fixed; then $C$, which is the symmetric difference of $C_{2}$ and $C_{2}^{\prime}$, is sign-fixed. In either case we have proved Claim 2.

The proof is now settled with two final cases.

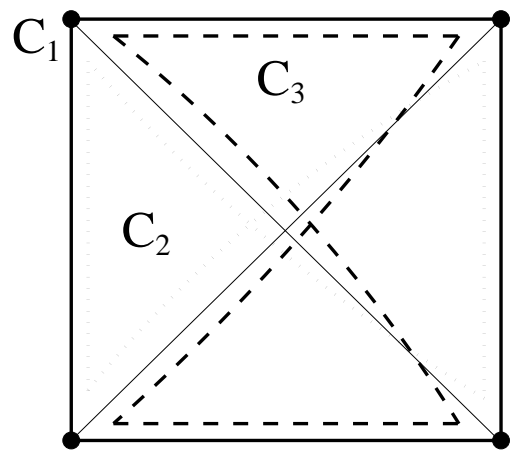

Figure 12: Decomposition when $k=4$.

Case 1: $\quad k=4$. Let $C_{1}$ be a circuit of length 4 in a prime graph $G$. If $G\left[V_{C_{1}}\right]$ is not complete then $G\left[V_{C_{1}}\right]$ contains a vertex of degree 2, so, by Claim 1, $C_{1}$ is sign-fixed. Thus we may suppose that $G\left[V_{C_{1}}\right]$ is complete. Let $C_{2}$ and $C_{3}$ be defined by Figure 12 . By Claim 2, one of $C_{1}$ and $C_{2}$ is sign-fixed. If $C_{1}$ is sign-fixed we are done, so suppose $C_{2}$ is sign-fixed. Similarly one of $C_{1}$ and $C_{3}$ are sign-fixed, so suppose $C_{3}$ is sign-fixed. However $C_{1}$ is the symmetric difference of $C_{2}$ and $C_{3}$, so $C_{1}$ is sign-fixed.

Case 2: $\quad k>4$. Let $C$ be a circuit of length $k$ in a prime graph $G$. If $C$ is decomposable or if $G\left[V_{C}\right]$ contains a vertex of degree 2 then $C$ is sign-fixed. Suppose then that $C$ is indecomposable and that every vertex in $G\left[V_{C}\right]$ has degree at least 3 . Let $e$ be a chord of $C$ such that the distance in $C$ between the ends of $e$ is minimum among all chords of 
$C$. Let $y_{1}, \ldots, y_{r}$ be the internal vertices of a shortest path in $C$ between the ends of $e$. Since each vertex in $V_{C}$ has degree at least 3 in $G\left[V_{C}\right]$, each $y_{i}$ must subtend at least one chord of $C$; let $e_{i}$ be a chord having $y_{i}$ as an end. The distance in $C$ between the ends of $e_{i}$ is at least the distance between the ends of $e$ in $C$, so $e_{i}$ must cross $e$. Since $C$ is indecomposable, there is at most one chord crossing $e$; therefore $r=1$. Furthermore $e_{1}$ and $e$ must be a tight crossing pair, so the other end of $e_{1}$ must also be adjacent to an end of $e$ in $C$. Therefore there are consecutive vertices $x_{1}, x_{2}, x_{3}, x_{4}$ of $C$ such that $x_{1}$ and $x_{3}$ are the ends of $e$, and $x_{2}$ and $x_{4}$ are the ends of $e_{1}$. Let $C^{\prime}$ be the circuit $x_{1}, x_{2}, x_{4}, x_{3}, x_{1}$; $C^{\prime}$ is sign-fixed since it has length 4. By Claim 2 at least one of $C$ and $C \Delta C^{\prime}$ is sign-fixed. If $C$ is sign-fixed we are done. Otherwise $C \Delta C^{\prime}$ is sign-fixed, so $C$ (which is the symmetric difference of $C \Delta C^{\prime}$ and $C^{\prime}$ ) is also sign-fixed. This completes the proof.

\section{Constructing a PU-orientation}

Let $G=(V, E)$ be a simple graph that admits a PU-orientation. In Section 2 we essentially described how to construct all PU-orientations of $G$ from a single PU-orientation. In this section, we outline a polynomial-time algorithm that provides the initial PU-orientation. By Proposition 2.1, we may assume that $G$ is prime.

We fix an arbitrary orientation $\vec{G}_{0}=\left(V, \vec{E}_{0}\right)$ of $G$. Thus orientations can be conve-

niently encoded by $(0,1)$-vectors indexed by $E$. Specifically, an orientation $\vec{G}$ is encoded by $x \in\{0,1\}^{E}$ where $x_{e}=0$ if and only if $\vec{G}$ and $\vec{G}_{0}$ concur in their orientation of $e$. Henceforth we refer to an orientation by its encoding.

Let $\mathcal{C}_{0}$ denote the set of edge sets of even circuits of $G$. Let $M$ be the incidence matrix of even circuits versus edges of $G$. That is, $M$ is a $(0,1)$-matrix with rows $\mathcal{C}_{0}$ and columns $E$ where, for $C \in \mathcal{C}_{0}$ and $e \in E$, the $(C, e)$ entry of $M$ is 1 if and only if $e \in C$. Let $v, v^{*} \in\{0,1\}^{E}$, where $v^{*}$ is a PU-orientation, and let $b=M v^{*}$. Then, by Theorem 1.1, $v$ is $\mathrm{PU}$ if and only if $v$ satisfies the binary matrix equation $M v=b$. Let $\mathcal{B}_{0} \subseteq \mathcal{C}_{0}$ be a basis of the even-circuit space (that is, the rowspace of $M$ over GF(2)). We now define, $M^{\prime}=M\left[\mathcal{B}_{0}, E\right]$ and $b^{\prime}=M^{\prime} v^{*}$. Then, $M^{\prime} v=b^{\prime}$ if and only if $M v=b$. Consequently, for $v \in\{0,1\}^{E}, v$ is $P U$ if and only if $M^{\prime} v=b^{\prime}$ over $G F(2)$. Our algorithm finds a PUorientation by solving the binary matrix equation $M^{\prime} v=b^{\prime}$. At this point there remain two obstacles in implementing the algorithm, namely:

(1) How can we find a basis for the even-circuit space efficiently?

(2) For an even circuit $C$, how can we compute $b_{C}$ efficiently (without knowing $v^{*}$ )?

Let $\mathcal{C}$ denote the set of edge sets of circuits of $G$. The circuit space (that is the rowspace, over $G F(2)$, of the circuit-edge incidence matrix of $G$ ) is the set of incidence vectors of eulerian subgraphs of $G$. Thus, by Proposition 3.7, there exists a basis $\mathcal{B} \subseteq \mathcal{C}$ of the circuit space that contains at most one odd circuit. For bipartite graphs this is trivial; for nonbipartite graphs such a basis can be constructed efficiently by making an ear decomposition of $G$ that begins with an odd circuit; we leave the details to the reader. Given such a basis of the circuit space, the even circuits form a basis of the even-circuit space. This answers (1). 
The second of the aforementioned problems is less trivial. However, our proof of Theorem 1.1 is essentially a recursive algorithm for computing $b_{C}$. The algorithm relies on the following strengthening of Proposition 3.6, whose proof is left to the reader.

Proposition 4.1 Let $C, C_{1}, \ldots, C_{k}$ be even circuits of $G$ such that $C=C_{1} \Delta \ldots \Delta C_{k}$. Then $b_{C}=b_{C_{1}}+\ldots+b_{C_{k}}$ modulo 2 .

Our algorithm immediately separates the cases where $|C|=4$ and $|C|>4$. However, in each case we must solve the subproblem given in Claim 2; precisely, the problem is as follows.

Subproblem: Let $C$ be an even circuit with consecutive vertices $x_{1}, \ldots, x_{4}$ such that $x_{1} x_{3}$ and $x_{2} x_{4}$ are chords, and let $C^{\prime}$ be the symmetric difference of $C$ and the circuit $x_{1}, x_{3}, x_{4}, x_{2}, x_{1}$. Find $b_{C}$ or $b_{C^{\prime}}$.

The algorithm for this subproblem comes directly from the proof of Claim 2 . We leave the details to the reader, and instead focus on the main algorithm.

Suppose that $|C|=4$. If $G\left[V_{C}\right]$ has a vertex of degree 2 , then $b_{C}$ can easily be computed using Lemma 2.2. Thus we assume $G\left[V_{C}\right]$ is complete, and is depicted in Figure 12. By using the subproblem twice, we determine two of $b_{C_{1}}, b_{C_{2}}, b_{C_{3}}$, and the third is obtained by their sum.

We now consider the case that $|C|>4$.

If $C$ is decomposable, then we can express $C$ as the symmetric difference of circuits $C_{1}, \ldots, C_{k}$, as described in Figures 5, 6 and 7 , such that $\left|C_{i}\right|<|C|$, for $i=1, \ldots, k$, and $\sum_{i=1}^{k}\left|C_{i}\right| \leq|C|+8$. Thus $b_{C}$ can be computed recursively as the sum of the $b_{C_{i}}$. The conditions on the sizes of these circuits maintains the efficiency of the algorithm. Henceforth we assume that $C$ is indecomposable.

Now suppose that $G\left[V_{C}\right]$ has a vertex $v$ of degree 2 . Let $w$ be a vertex adjacent to $v$. Note that changing the orientation of an edge in $\vec{G}_{0}$ has a predictable effect on $b_{C}$. We change the orientation $\vec{G}_{0}$ so that we have the following property: Each edge xy with $x \in N(w)$ and $y \in N(v)$ is oriented with its head being a neighbour of $v$ and its tail being a neighbour of $w$. (Note that we allow $x=v$ and $y=w$.) We leave it to the reader to check that this property ensures that partial pivoting on $\{v, w\}$ in the adjacency matrix of $\vec{G}_{0}$ yields a $(0, \pm 1)$-matrix. Let $\vec{G}_{0}^{\prime}$ be the oriented graph obtained by this partial pivot, and let $G^{\prime}$ be the graph obtained by performing a partial pivot on $v w$ in $G$. Note that $\vec{G}_{0}^{\prime}$ is an orientation of $G^{\prime}$; also $C$ is a circuit of $G^{\prime}$ and $b_{C}$ is unaffected by the pivot. However, the partial pivot added an even chord to $C$, making $C$ decomposable. Henceforth we may assume that $G\left[V_{C}\right]$ has no vertex of degree 2 .

By the assumptions on $C$, we can find consecutive vertices $x_{1}, x_{2}, x_{3}, x_{4}$ of $C$ such that $x_{1} x_{3}$ and $x_{2}, x_{4}$ are chords. Let $C_{0}$ denote the circuit $x_{1}, x_{2}, x_{4}, x_{3}, x_{1}$. Since $C$ is indecomposable, $x_{1} x_{4}$ is not a chord. Thus $b_{C_{0}}$ can be computed easily by Lemma 2.2 . Let $C^{\prime}$ be the symmetric difference of $C$ and $C_{0}$. We now use the subproblem to find $b_{C}$ or $b_{C^{\prime}}$. Thus we know two of $b_{C_{0}}, b_{C}, b_{C^{\prime}}$, their sum gives us the third. This completes the algorithm.

\section{ACKNOWLEDGMENTS}

The authors wish to thank M. Loebl and W. Hochstättler for stimulating discussions. 


\section{References}

[1] A. Bouchet. Greedy algorithm and symmetric matroids. Math. Programming, 38:147$159,1987$.

[2] A. Bouchet. Reducing prime graphs and recognizing circle graphs. Combinatorica, 7:243-254, 1987.

[3] A. Bouchet. Unimodularity and circle graphs. Discrete Math., 66:203-208, 1987.

[4] A. Bouchet. Representability of $\Delta$-matroids. Colloquia Societatis Janos Bolyia, 52:167-182, 1988.

[5] A. Bouchet. Matroid connectivity and fundamental graphs. In Proceedings of the Twenty-Second Southeastern Conference on Combinatorics, Graph Theory and Computing, 1991.

[6] A. Bouchet. A characterization of unimodular orientations of simple graphs. J. Combin. Theory Ser. B, 56:45-54, 1992.

[7] A. Bouchet. Circle graph obstructions. J. Combin. Theory Ser. B, 60:107-144, 1994.

[8] P. Camion. Caractérisation des matrices unimodulaires. Cahiers Centre Études Rech. Opér., 5:181-190, 1963.

[9] W. H. Cunningham. Decomposition of directed graphs. SIAM J. Algebra Discrete Math., 32:214-228, 1982.

[10] H. de Fraysseix. A characterization of circle graphs. European J. Combin., 5:223-238, 1984.

[11] J. F. Geelen. A generalization of Tutte's characterization of totally unimodular matrices. In preparation.

[12] J. F. Geelen. Matchings, matroids, and unimodular matrices. PhD thesis, University of Waterloo, 1995.

[13] J. F. Geelen, A. M. H. Gerards, and A. Kapoor. The excluded minor characterization of $\mathrm{GF}(4)$-representable matroids. In preparation.

[14] A. M. H. Gerards. A short proof of Tutte's characterization of totally unimodular matrices. Linear Algebra Appl., 114/115:207-212, 1989.

[15] C. D. Godsil. Algebraic Combinatorics. Chapman and Hall, 1993.

[16] I. B.-A. Hartman. The Cycle Space of a Graph. Master's thesis, University of Waterloo, 1982.

[17] J. Kahn. On the uniqueness of matroid representations over GF(4). Bull. London Math. Soc., 20:5-10, 1988. 
[18] A. Kotzig. Quelques remarques sur les transformations $\kappa$. séminaire Paris, 1977.

[19] J. R. Stembridge. Nonintersecting paths, pfaffians, and plane partitions. Advances in Math., 83:96-131, 1990.

[20] W. T. Tutte. A homotopy theorem for matroids, I, II. Trans. Amer. Math. Soc., $88: 144-174,1958$.

[21] W. T. Tutte. Lectures on matroids. J. Res. Nat. Bur. Standards Sect. B, 69b:1-47, 1965. 